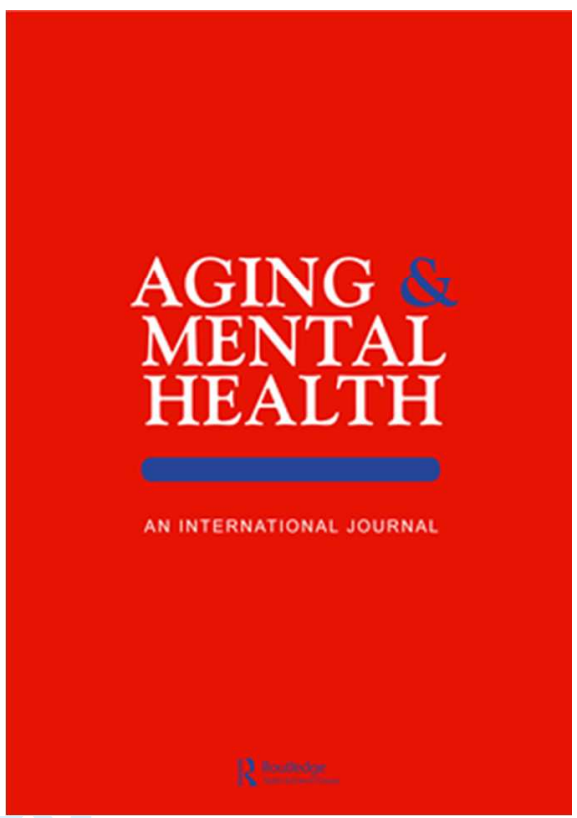

Theory of Mind and Social Relationships in Older Adults: the Role of Social Motivation

\begin{tabular}{|r|l|}
\hline Journal: & Aging and Mental Health \\
\hline Manuscript ID & CAMH-2015-0280.R1 \\
\hline Manuscript Type: & Original Article \\
\hline Keywords: & theory of mind, social relationships, aging, social motivation \\
\hline \multicolumn{2}{|c}{} \\
\hline
\end{tabular}

SCHOLARONE ${ }^{\mathrm{m}}$

Manuscripts 
Abstract

\title{
Theory of Mind and Social Relationships in Older Adults: the Role of Social Motivation
}

\begin{abstract}
Aging \& Mental Health
Objectives: Previous research has shown that individual differences in Theory of Mind (ToM) are crucial for people's social relationships. However, very few studies have investigated this issue in aging. The present study was designed to fill this gap and examine the associations between ToM and social relationships in elderly adults. In doing so, this study considered people's relationships with their relatives and friends and examined the possible moderating role of social motivation.
\end{abstract}

Method: The study involved 53 healthy older adults $(\mathrm{M}$ age $=67.91 ; \mathrm{SD}=6.93$; range: 60-85 years). All participants were tested collectively during a 2-hr session and completed a demographic questionnaire as well as a battery of tests assessing verbal ability (vocabulary, and word fluency), ToM, and social relationships. They also answered a social motivation question.

Results: Results showed that individual differences in older people's ToM were, overall, significantly associated with those in relationships with friends, but not relatives. In addition, the Hayes moderating procedure showed that individual differences in ToM were related to those in friendships only for those people who had a high or medium level of social motivation.

Conclusion: These findings underline the importance of motivation in guiding the use of ToM in everyday social interactions.

Keywords: theory of mind; social relationships; aging; social motivation. 


\section{Introduction}

Social relationships represent a universal human need (Maslow, 1943); across ones’ life span, individuals search for social contact (Baumeister \& Leary, 1995) and suffer due to social isolation (Berkman, Glass, Brissette, \& Seeman, 2000). The term "social relationship" is very general and encompasses at least two dimensions: the structure and the function (Cohen \& Wills, 1985; Lubben \& Gironda, 2003). The structure refers to objective traits, such as size and frequency of contacts. The function reflects the quality of the relationship and refers to closeness and social support. A considerable body of research has documented the benefits of both these aspects on elderly people's physical health and cognitive functioning (Béland, Zunzunegui, Alvarado, Otero, \& del Ser, 2005; Holt-Lunstad, Smith, \& Layton, 2010). Given the importance of social life in aging, there is a need for an investigation of the factors that may impact social relationships. In the present study we focused on Theory of Mind (ToM).

ToM is defined as the awareness that mental states (thoughts, beliefs, emotions) guide social behaviour (Premack \& Woodruff, 1978) and allows people to predict, explain, and even affect others' social behaviour. Studies on clinical conditions (Brüne, Abdel-Hamid, Lehmkämper, \& Sonntag, 2007; Peterson, Garnett, Kelly, \& Attwood, 2009) and typical developing children (Slaughter, Imuta, Peterson, Henry, 2015) indicate that ToM is crucial for positive social interactions. ToM helps people to build and maintain positive social relationships as it permits the understanding of complex social scenarios (Stone, BaronCohen, \& Knight, 1998; McKinnon \& Moscovitch, 2007), others’ needs (Caputi, Lecce, Pagnin, \& Banerjee, 2012) and reduces the risk of misunderstanding (Hughes \& Leekam, 2004).

To date we know very little about the role of ToM skills in older people's social relationships. This issue is relevant both theoretically and practically. Theoretically, it is 
important to know whether the link between these two constructs persists across life or not.

On one hand, it is possible that the association between ToM and social relationships is constant across life span (see the paragraph below). On the other hand, it is also plausible that the link between social relationships and ToM is attenuated during life and that social relationships in aging rely on learned behavioral strategies (Ickes \& Simpson, 2007; Blanchard-Fields, 2007), acquired social knowledge (i.e., appreciation of contexts and goals), and greater social expertise (Hess, Osowski, \& Leclerc, 2005) rather than on mental states understanding. From a practical point of view, knowing antecedents of social relationships in aging will help researchers to design effective programs to promote adequate social functioning, that is vital for wellbeing (Pinquart \& Sörensen, 2000). To date, only two studies have directly investigated the associations between ToM and social life in aging. The first is the study by Bailey, Henry, and Von Hippel (2008) that focused on cognitive empathy, defined as the ability to understand others' mental states ${ }^{1}$, and social functioning, defined as the level of participation in social activities that one generally does in the company of others. Results showed that the age-related decline in social functioning could be due to a reduction in socio-cognitive skills. In the second study, Yeh (2013) examined the role of ToM and executive functions in explaining social intelligence in elderly people. Social intelligence was defined as a group of skills (i.e., social skills, social context awareness) that allows people to understand themselves and others and to successfully interact with others. Analyses showed that ToM (as indexed by faux pas recognition) was significantly related with social skills and that the link between social intelligence and ToM was stronger than the one between social intelligence and executive functioning. Overall, these studies suggest that the link between ToM and social relationships persists in old age.

\footnotetext{
${ }^{1}$ The construct of cognitive empathy has been equated with Theory of Mind (Blair, 2005).
} 
In the present study, we followed up this line of investigation and extended it in two ways. Firstly, we looked at two social relationships: those with relatives and friends.

Secondly, we considered the role of social motivation. Indeed, while studies cited above provide some evidence for the existence of an association between ToM and social relationships in elderly people, the results showed a variability in the strength of this association. Thus, it is likely that the link between ToM and social relationships in aging is moderated by other variables.

We focused on relationships with friends and relatives as, with increasing age, people carefully select activities that are emotionally meaningful (Hendricks \& Cutler, 2004). As a result of this change, older adults tend to interact with close friends and relatives to a greater extent than do younger adults (Fung, Carstensen, \& Lutz, 1999) and relationships with relatives and close friends are crucial for successful aging (Hertzog, Kramer, Wilson, \& Lindenberger, 2008). Because both the quantity and the quality of social relationships play a specific role in predicting wellbeing and quality of life in elderly population (Rafnsson, Shankar, \& Steptoe, 2015), we considered both these aspects in order to have a comprehensive measure of relationships with friends and relatives.

As anticipated, in the present study we also considered the possible moderating role of social motivation in the association between ToM and social relationships. The construct of motivation, defined as the reasons underpinning people's thoughts and actions (Weiner, 1992), is particularly important in aging. As outlined by the Selective Engagement Hypothesis (Hess, 2006), elderly people tend to either avoid cognitively demanding activities or to engage themselves in activities that are meaningful to them and are, thus, worth the effort (Hess, Quinn, \& Ennis, 2013). With respect to ToM, a number of studies involving dual tasks (Bull, Philipps, \& Conway, 2008; German \& Hehman, 2006) and matched control tasks (Phillips, Bull, Allen, Insch, Burr, \& Ogg, 2011; Sullivan \& Ruffman, 2004) has shown 
that ToM is cognitively demanding for elderly adults. Thus, it seems likely that older adults use their ToM only if motivated. Consistent to this interpretation, Zhang and colleagues showed that motivation modulates the effect of age differences in perspective taking tasks (Zhang, Fung, Stanley, Isaacowitz, \& Ho, 2013). They took as their premise that older adults increasingly prefer emotionally meaningful goals, such as maintaining close social relationships, and ran two studies manipulating participants' motivation by shaping perceived closeness between participants and the experimenter. Results showed that when dealing with someone to whom they feel closer (and, presumably, for whom they are more motivated), older adults performed equally as well as the younger adults. These results, thus, suggest that what is important is not only to possess a good understanding of others' mental states but also to be motivated to use this understanding in everyday social relationships. Following this line of interpretation, we expected that the relation between ToM and social relationships holds up as significant only for people with high levels of social motivation. In the present paper we measured a specific aspect of social motivation, namely the importance of being liked by others. We chose this aspect because being liked by others is a crucial aspect of social motivation (Bergsieker, Shelton, \& Richeson, 2010). Sociological studies have, indeed, shown how the human need to be involved in social relationships makes people constantly worried about how they are perceived about others (Baumeister \& Leary, 1995) and motivates them to act in order to minimize the possibility of being refused (Leary, Tambor, Terdal, \& Downs, 1995). Given this, we expected that people who think that being liked by others is important were more likely to use their ToM to build and maintain positive social relationships.

The main aim of the present study was to examine the association between older adults' ToM and their relationships with relatives and friends and to consider the moderating role of social motivation. In order to do that we controlled for fluid and crystallized 
intelligence given their relations with both ToM (Charlton, Barrick, Markus, \& Morris, 2009; Moran, 2013) and social functioning (Hughes, Andel, Small, Borenstein, \& Mortimer, 2008; Keller-Cohen, Fiori, Toler \& Bybee, 2006). Given existing results (Bailey et al., 2008; Yeh, 2013), we expected ToM to be significantly related with the relationships that people have with both relatives and friends. In addition, since ToM is cognitively demanding, we expected that the link between ToM and social relationships (with both relatives and friends) held up as significant only for those people with high social motivation.

\section{Method}

\section{Participants}

Participants were 53 (28.3\% men) older adults (age: $M=67.91, S D=6.93$, range 60 85 ; years of education: $M=13.28, S D=3.75$, range5-20) recruited through the local branch of the University of Third Age in northern Italy. All subjects were volunteers and lived independently, were reasonably fit and healthy, and had active social and cognitive lifestyles. Italian was the first language of all participants. Prior to testing, participants filled out a general demographic questionnaire to exclude diagnosis of dementia or neurological disorders. We administered the CES-D (Fava, 1983) to make sure no participants had depression. None of the participants was excluded on the basis of the above criteria. The participants' demographic characteristics are reported in Table 1. This research was approved by the Ethics Committee of the Department of Brain and Behavioural Sciences, University of Pavia. All participants provided written informed consent to participate in the study.

\section{Materials and Procedure}

Participants were tested collectively during a 2-hr session in which they completed a demographic questionnaire and a battery of tests assessing vocabulary, word fluency, social motivation, ToM, and social relationships. 
Control variables. A Vocabulary test (drawn by Primary Mental Abilities test;

Thurstone \& Thurstone, 1963) was included in the study as a control variable of crystallized intelligence. This test requires to identify the correct synonym of 50 target words choosing between 4 alternatives within 8 minutes. We also administered a Word Fluency subtest asking participants to generate as many words as possible, according to a phonemic rule (i.e., words beginning with "s") during a 90 second period for each trial (Dahlin, Nyberg, Backman, \& Neely, 2008).

Social Motivation. It was measured via the following item from the Social Sensitivity subscale of the Social Skills Inventory (SSI; Riggio, 1986): “It is very important that other people like me". Participants were asked to indicate on a 5-point-Likert scale the extent to which the description given in the item applied to them.

ToM assessment. It was assessed using 4 ToM stories of the Strange Stories task (White, Hill, Happé, \& Frith, 2009). The stories consisted of two double bluff and two misunderstanding. According to the scoring procedure (White et al., 2009), participants' answers to each story were scored as a 2 for full and explicit answer consisting in a mental state explanation, 1 for partially correct answer, and 0 for an incorrect answer. Two raters independently coded $25 \%$ of the responses at pre-and post-test. The degree of accordance, established with Cohen's Kappa, was good $(\mathrm{k}=.87)$. The possible score range was $0-8$.

Social Relationships assessment. It was assessed using the revised version of the Lubben Social Network Scale-Revised (LSNS-R; Lubben \& Gironda, 2003; 2004). It measures people's social relationships with relatives (6 items) and friends (6 items). Each subscale (relatives and friends) contains items that measure size, closeness, and frequency of contact within respondent's social network. Participants responded on a 6-point scale from 0 (none/never) to 5 (nine or more/always). An index for each domain (relatives vs. friends) was 
obtained with higher scores representing higher social relationships (relatives, $\alpha=.75$; friends, $\alpha=.79$ ). The possible score range was $0-30$ for each scale.

\section{Results}

The pattern of associations between variables are reported in Table 1. As it can be seen, vocabulary and fluency were significantly associated with one another. In addition, vocabulary significantly correlated with ToM, whereas word fluency was correlated with friendships. Regarding the associations between focus variables, our data showed that older people's ToM is significantly associated with friendships but not relationships with relatives. Crucially, the association between friendships and ToM remained significant when vocabulary and word fluency were taken under control, $r=.32, p=.02$. Social motivation was not significantly related with either ToM or social relationships.

\section{Insert Table 1 about here}

In order to test whether social motivation moderates the association between ToM and friendships, we run a three-step multiple linear regression analysis with friendships as dependent variable. Firstly, we entered vocabulary and word fluency at first step, as control variables. At the second step we entered ToM and social motivation. Finally, we added the interaction term ToM X social motivation. Results (see Table 2) showed that Step 1 and 2 explained only marginal amounts of variance $(F \geq 2.46, p \leq .08)$. At Step 3 the equation was significant, $R^{2}=.25, F(5,45)=2.95, p=.02$. Crucially, the interaction term explained a significant amount of unique variance in friendships, $\Delta R^{2}=.07, \Delta F(1,45)=4.22, p=.04$.

Insert Table 2 about here

Simple slopes analysis indicated that ToM was positively related to friendships at a high (at exactly $+1 S D$ above the mean), $b=.58, p=.005$, and medium, $b=.27, p=.049$, level of social motivation. No relations between ToM and friendships were found for low levels (at exactly -1 SD below the mean) of social motivation, $b=.04$, n.s. (see Figure 1). 
Insert Figure 1 about here

\section{Discussion}

Our first important finding is that individual differences in older people's ToM are significantly associated with those in relationships with friends, but not relatives. This result, despite being unexpected, is interesting as it shows that the link between ToM and the social sphere is specific, and not general. We argue that this result can be explained by taking into account the nature of the two relationships considered. Indeed, despite their comparable role, relationships between friends and relatives differ on many dimensions (Laursen \& Bukowski, 1997). Perhaps, the most important is that while family ties are predetermined, friendships are created and maintained on a voluntary basis (Antonucci \& Akiyama, 1995).

Consequently, older adults may have generally less opportunities to use their social skills in their family relations, because these relationships have a strong normative component (Litwak, 1981). On the contrary, people are likely to exert more energy to maintain their relationships with friends than relatives (Roberts \& Dunbar, 2011).

The second important result of the present study is the moderating role of social motivation. Here, our data showed that individual differences in ToM were related to those in friendships only for people who had a high or medium level of social motivation. This suggests that, in order to have good and supportive friendships, elderly people need both to have ToM skills and to be motivated to put these skills into use for social purposes. This result fits with the view of ToM as a socially neutral tool that can or cannot be used. Accordingly, having an ability (marked by scores on experimental tasks) is not sufficient per se, as one also needs to have the motivation to use this understanding to guide everyday interactions with others (Begeer, Malle, Nieuwland, \& Keysar, 2010; Klein \& Hodges, 2001).

On a broad note, our finding that ToM has to be supported by high social motivation to guarantee positive relationships fits the Selective Engagement Hypothesis (Hess, 2006). It 
does not argue for stability of deliberative skills with age, but rather claims that these skills are put into action in a flexible way to support adequate functioning in real life.

The present study can be considered as a pioneering investigation that, we believe, has important implications in the field of intervention programs. Our results suggest that, in order to have positive effects on real life, researchers should improve ToM abilities and, at the same time, social motivation. Some researchers have shown that it is possible to enhance ToM in healthy aging people (Cavallini, Bianco, Bottiroli, Rosi, Vecchi, \& Lecce, 2015; Lecce, Bottiroli, Bianco, Rosi, \& Cavallini, 2015). These training programs should be revised in the light of the results of the present study.

Although the present study examined an important issue from an innovative angle, it has some limitations that deserve attention. First, social motivation was investigated with only one item from a questionnaire. We chose this item as it reflects particular attention towards other people and an inclination to perform socially competent behaviours. However, a more comprehensive and robust measure is needed. Second, the number of participants is relatively low and replication with a bigger sample is required. It would also be useful to expand our results using more objective assessment of social functioning. Further research is also needed to investigate whether there are any (and which) factors that may moderate the link between ToM and social factors, such as cultural background. For instance, Cavallini and colleagues (Cavallini, Bottiroli, Fastame, \& Hertzog, 2013) have demonstrated how other metacognitive components (i.e., cognitive beliefs) may change across cultures and differently affect cognitive performance. Finally, experimental or longitudinal design could yield a deeper understanding of the causal link between ToM and social relationships in aging.

\section{Conflict of interest}

The authors have no conflicts of interest to disclose. 


\section{References}

Antonucci, T. C., \& Akiyama, H. (1995). Convoys of social relations: Family and friendships within a life span context. In R. Blieszner \& V. H. Bedford (Eds.), Handbook of aging and the family (pp. 355-371). Westport, CT, US: Greenwood Press/Greenwood Publishing Group.

Bailey, P. E., Henry, J. D., \& von Hippel, W. (2008). Empathy and social functioning in late adulthood. Aging and Mental Health, 12, 499-503. doi:10.1080/13607860802224243

Baumeister, R., \& Leary, M. R. (1995). The need to belong: Desire for interpersonal attachments as a fundamental human motivation. Psychological Bulletin, 117, 497529. doi:10.1037/0033-2909.117.3.497

Begeer, S., Malle, B. F., Nieuwland, M. S., \& Keysar, B. (2010). Using theory of mind to represent and take part in social interactions: Comparing individuals with highfunctioning autism and typically developing controls. European Journal of Developmental Psychology, 7, 104-122. doi:10.1080/17405620903024263

Béland, F., Zunzunegui, M. V., Alvarado, B., Otero, A., \& del Ser, T. (2005). Trajectories of cognitive decline and social relations. The Journals of Gerontology Series B: Psychological Sciences and Social Sciences, 60, 320-330. doi:10.1093/geronb/60.6.P320

Bergsieker, H. B., Shelton, J. N., \& Richeson, J. A. (2010). To be liked versus respected: Divergent goals in interracial interactions. Journal of Personality and Social Psychology, 99, 248-264. doi:10.1037/a0018474

Berkman, L. F., Glass, T., Brissette, I., \& Seeman, T. E. (2000). From social integration to health: Durkheim in the new millennium. Social Science \& Medicine, 51, 843-857. doi:10.1016/S0277-9536(00)00065-4 
Blair, R. J. R. (2005). Responding to the emotions of others: Dissociating forms of empathy through the study of typical and psychiatric populations. Consciousness and Cognition, 14, 698-718. doi:10.1016/j.concog.2005.06.004

Blanchard-Fields, F. (2007). Everyday problem solving and emotion: An adult developmental perspective. Current Directions in Psychological Science, 16, 26-31. doi:10.1111/j.1467-8721.2007.00469.x

Brüne, M., Abdel-Hamid, M., Lehmkämper, C., \& Sonntag, C. (2007). Mental state attribution, neurocognitive functioning, and psychopathology: What predicts poor social competence in schizophrenia best? Schizophrenia Research, 92, 151-159. doi:10.1016/j.schres.2007.01.006

Bull, R., Phillips, L. H., \& Conway, C. A. (2008). The role of control functions in mentalizing: Dual-task studies of theory of mind and executive function. Cognition, 107, 663-672. doi:10.1016/j.cognition.2007.07.015

Caputi, M., Lecce, S., Pagnin, A., \& Banerjee, R. (2012). Longitudinal effects of theory of mind on later peer relations: The role of prosocial behaviour. Developmental Psychology, 48, 257-270. doi:10.1037/a0025402

Cavallini, E., Bianco, F., Bottiroli, S., Rosi, A., Vecchi, T., \& Lecce, S. (2015). Training for generalization in Theory of Mind: A study with older adults. Frontiers in Psychology, 6, 1123. doi:10.3389/fpsyg.2015.01123

Cavallini, E., Bottiroli, S., Fastame, M. C., \& Hertzog, C. (2013). The role of age and culture on personal and general beliefs about memory. Journal of Aging Studies. 27, 71-81. doi:10.1016/j.jaging.2012.11.002

Charlton, R. A., Barrick, T. R., Markus, H. S., \& Morris, R. G. (2009). Theory of mind associations with other cognitive functions and brain imaging in normal aging. Psychology and Aging, 24, 338-348. doi:10.1037/a0015225 
Cohen, S., \& Wills, T. A. (1985). Stress, social support, and the buffering hypothesis. Psychological Bulletin, 98, 310-357. doi:10.1037/0033-2909.98.2.310

Dahlin, E., Nyberg, L., Bäckman, L., \& Neely, S. A. (2008). Plasticity of executive functioning in young and older adults: Immediate training gains, transfer, and longterm maintenance. Psychology and Aging, 23, 720-730. doi:10.1037/a0014296

Fava, G. A. (1983). Assessing depressive symptoms across cultures: Italian validation of the CES-D self-rating scale. Journal of Clinical Psychology, 39, 249-251. doi:10.1002/1097-4679(198303)39:2<249::AID-JCLP2270390218>3.0.CO;2-Y

Fung, H. H., Carstensen, L. L., \& Lutz, A. (1999). The influence of time on social preferences: Implications for life-span development. Psychology and Aging, 14, 595604. doi:10.1037/0882-7974.14.4.595

German, T. P., \& Hehman, J. A. (2006). Representational and executive selection resources in 'theory of mind': Evidence from compromised belief-desire reasoning in old age. Cognition, 10, 129-152. doi:10.1016/j.cognition.2005.05.007

Hendricks, J., \& Cutler, S. J. (2004). Volunteerism and socioemotional selectivity in later life. The Journals of Gerontology Series B: Psychological Sciences and Social Sciences, 59B, 251-257. doi:10.1093/geronb/59.5.S251

Hertzog, C., Kramer, A. F., Wilson, R. S., \& Lindenberger, U. (2008). Enrichment effects on adult cognitive development: Can the functional capacity of older adults be preserved and enhanced? Psychological Science in the Public Interest, 9, 1-65. doi:10.1111/j.1539-6053.2009.01034.x

Hess, T. M. (2006). Adaptive aspects of social cognitive functioning in adulthood: Agerelated goal and knowledge influences. Social Cognition, 24, 279-309. doi:10.1521/soco.2006.24.3.279 
Hess, T. M., Osowski N. L., \& Leclerc C.M. (2005). Age and experience influences on the complexity of social inferences. Psychology and Aging. 20, 447-459. doi:10.1037/0882-7974.20.3.447

Hess, T. M., Queen, T. L., \& Ennis, G. E. (2013). Age and self-relevance effects on information search during decision-making. The Journals of Gerontology, Series B: Psychological Sciences and Social Sciences, 65B, 703-711. doi:10.1093/geronb/gbs108

Holt-Lunstad, J., Smith, T. B., \& Layton, J. B. (2010). Social relationships and mortality risk: a meta-analytic review. PLoS medicine, 7, e1000316. doi:10.1371/journal.pmed.1000316

Hughes, C., \& Leekam, S. (2004). What are the links between theory of mind and social relations? Review, reflections and new directions for studies of typical and atypical development. Social Development, 13, 590-619. doi:1 0.1111/j.14679507.2004.00285.x

Hughes, T. F., Andel, R., Small, B. J., Borenstein, A. R., \& Mortimer, J. A. (2008). The association between social resources and cognitive change in older adults: evidence from the Charlotte County Healthy Aging Study. The Journals of Gerontology Series B: Psychological Sciences and Social Sciences, 63, 241-244. doi:10.1093/geronb/63.4.p241

Ickes, W., \& Simpson, J. A. (2007). Motivational aspects of empathic accuracy. In G. J. O. Fletcher \& M. S. Clark (Eds.), Blackwell Handbook of Social Psychology: Interpersonal Processes (pp. 229-249). Malden, MA: Blackwell Publishers Ltd. doi:10.1002/9780470998557.ch9 
Keller-Cohen, D., Fiori, K., Toler, A., \& Bybee, D. (2006). Social relations, language and cognition in the 'oldest old'. Ageing and Society, 26, 585-605. doi: $10.1017 / \mathrm{S} 0144686 \mathrm{X} 06004910$

Klein, K. J., \& Hodges, S. D. (2001). Gender differences, motivation, and empathic accuracy: When it pays to understand. Personality and Social Psychology Bulletin, 27, 720-730. doi: $10.1177 / 0146167201276007$

Laursen, B., \& Bukowski, W. M. (1997). A developmental guide to the organisation of close relationships. International Journal of Behavioral Development, 21, 747-770. doi:10.1080/016502597384659

Leary, M. R., Tambor, E. S., Terdal, S. K., \& Downs, D. L. (1995). Self-esteem as an interpersonal monitor: The sociometer hypothesis. Journal of Personality and Social Psychology, 68, 518-530. doi:0.1037/0022-3514.68.3.518

Lecce, S., Bottiroli, S., Bianco, F., Rosi, A., \& Cavallini, E. (2015). Training older adults on theory of mind: Transfer on metamemory. Archives of Gerontology and Geriatrics, 60, 217-226. doi:10.1016/j.archger.2014.10.001

Litwak, E. (1981). The modified extended family, social networks, and research continuities in aging. New York: Columbia University Center for Social Sciences.

Lubben, J. \& Gironda, M. (2003). Centrality of social ties to the health and well-being of older adults. In Berkman, B. \& Harootyan, L. (Eds.). Social work and health care in an aging society (pp. 319-350). New York: Springer.

Lubben, J. \& Gironda, M. (2004). Measuring social networks and assessing their benefits. In Phillipson, C., Allan, G., \& Morgan, D. (Eds.). Social networks and social exclusion: Sociological and policy perspectives. Hant, England: Ashgate Publishing Limited.

Maslow, A. H. (1943). A theory of human motivation. Psychological Review, 50, 370-396. doi: $10.1037 / \mathrm{h} 0054346$ 
McKinnon, M. C., \& Moscovitch, M. (2007). Domain-general contributions to social reasoning: Theory of mind and deontic reasoning re-explored. Cognition, 102, 179218. doi:10.1016/j.cognition.2005.12.011

Moran, J. M. (2013). Lifespan development: The effects of typical aging on theory of mind. Behavioural Brain Research, 237, 32-40. doi:10.1016/j.bbr.2012.09.020

Peterson, C. C., Garnett, M., Kelly, A., \& Attwood, T. (2009). Everyday social and conversation applications of theory-of-mind understanding by children with autismspectrum disorders or typical development. European Child and Adolescent Psychiatry, 18, 105-115. doi:10.1007/s00787-008-0711-y

Phillips, L. H., Bull, R., Allen, R., Insch, P., Burr, K., \& Ogg, W. (2011). Lifespan aging and belief reasoning: Influences of executive function and social cue decoding. Cognition, 120, 236-247. doi:10.1016/j.cognition.2011.05.003

Pinquart, M., \& Sörensen, S. (2000). Influences of socioeconomic status, social network, and competence on subjective well-being in later life: A meta-analysis. Psychology and Aging, 15, 187-224. doi:10.1037//0882-7974.15.2.187

Premack, D., \& Woodruff, G. (1978). Does the chimpanzee have a theory of mind? Behavioral and Brain Sciences, 1, 515-526. doi:10.1017/S0140525X00076512

Rafnsson, S. B., Shankar, A., \& Steptoe, A. (2015). Longitudinal Influences of Social Network Characteristics on Subjective Well-Being of Older Adults Findings From the ELSA Study. Journal of Aging and Health, 27, 919-934. doi: $10.1177 / 0898264315572111$

Riggio, R. E. (1986). Assessment of basic social skills. Journal of Personality and Social Psychology, 51, 649-660. doi:10.1037/0022-3514.51.3.649 
Roberts, S. G. B., \& Dunbar, R. I. M. (2011). The costs of family and friends: An 18-month longitudinal study of relationship maintenance and decay. Evolution and Human Behavior, 32, 186-197. doi:10.1016/j.evolhumbehav.2010.08.005

Slaughter, V., Imuta, K., Peterson, C. C., \& Henry, J. D. (2015). Meta-Analysis of Theory of Mind and Peer Popularity in the Preschool and Early School Years. Child Development. doi:10.1111/cdev.12372

Stone, V. E., Baron-Cohen, S., \& Knight, R. T., (1998). Frontal lobe contributions to theory of mind. Journal of Cognitive Neuroscience 10, 640-656. doi:10.1162/089892998562942

Sullivan, S., \& Ruffman, T., (2004). Social understanding: How does it fare with advancing years? British Journal of Psychology, 95, 1-18. doi:10.1348/000712604322779424

Thurstone, T. G., \& Thurstone, L. L. (1963). Primary mental ability. Chicago, IL: Science Research Associates.

Yeh, Z. T. (2013). Role of theory of mind and executive function in explain social intelligence: A structural equation modelling approach. Aging \& Mental Health, 17, 527-534. doi:10.1080/13607863.2012.758235

Weiner, B. (1992). Human motivation: Metaphors, theories and research. Newbury Park, CA: Sage Publications.

White, S., Hill, E., Happé, F. G. E., \& Frith, U. (2009). Revisiting the strange stories: Revealing mentalizing impairments in autism. Child Development, 80, 1097-1117. doi:10.1111/j.1467-8624.2009.01319.x.

Zhang, X., Fung, H. H., Stanley, J. T., Isaacowitz, D. M., \& Ho, M. Y. (2013). Perspective taking in older age revised: A motivational perspective. Developmental Psychology, 49, 1848-1858. doi:10.1037/a0031211 
Table 1. Means (and standard deviation) of measured variables and correlations between control and focus variables.

\begin{tabular}{|c|c|c|c|c|c|c|}
\hline & Vocabulary & $\begin{array}{c}\text { Word } \\
\text { Fluency }\end{array}$ & ToM & $\begin{array}{c}\text { Relatives } \\
\text { Relationships }\end{array}$ & Friendships & $\begin{array}{c}\text { Social } \\
\text { Motivation }\end{array}$ \\
\hline Vocabulary & & $.57 * * *$ & $.29^{*}$ & -.07 & .06 & -.18 \\
\hline Word Fluency & & - & .16 & .06 & $.29 *$ & -.09 \\
\hline ToM & & & - & -.04 & $.30 *$ & .13 \\
\hline Relatives Relationships & & & & - & $.51 * *$ & -.02 \\
\hline Friendships & & & & & - & -.02 \\
\hline Social Motivation & & & & & & - \\
\hline Means (SD) & $45.49(4.87)$ & $40.98(10.96)$ & $5.58(1.64)$ & $19.21(4.64)$ & $16.68(4.17)$ & $3.12(1.32)$ \\
\hline Actual range & $25-50$ & $15-59$ & $2-8$ & $9-29$ & $5-24$ & $1-5$ \\
\hline
\end{tabular}


Table 2. Hierarchical Multiple Regression analysis investigating interaction effect on friendships.

\begin{tabular}{|c|c|c|c|c|c|c|c|c|c|}
\hline \multirow[b]{2}{*}{ Predictor } & \multicolumn{3}{|c|}{ Step 1} & \multicolumn{3}{|c|}{ Step 2} & \multicolumn{3}{|c|}{ Step 3} \\
\hline & $B$ & $S E$ & $\beta$ & $B$ & $S E$ & $\beta$ & $B$ & $S E$ & $\beta$ \\
\hline Vocabulary & -.13 & .16 & -.14 & -.22 & .16 & -.24 & -.27 & .16 & $-.29^{+}$ \\
\hline Word Fluency & .36 & .16 & $.38^{*}$ & .36 & .15 & $.38^{*}$ & .35 & .15 & $.37 *$ \\
\hline ToM & & & & .28 & .14 & $.29 *$ & .28 & .13 & $.29 *$ \\
\hline Social Motivation & & & & -.05 & .13 & -.05 & -.07 & .13 & -.08 \\
\hline ToM x Social & & & & & & & .31 & .15 & $.27^{*}$ \\
\hline \multicolumn{10}{|l|}{ Motivation } \\
\hline$R^{2}$ & & .10 & & & .18 & & & .25 & \\
\hline$F$ for change in $R^{2}$ & & $2.70^{+}$ & & & 2.10 & & & $4.22 *$ & \\
\hline
\end{tabular}


Figure 1. Simple slopes of ToM at low (-1 SD), medium, and high (+1 SD) Social Motivation.

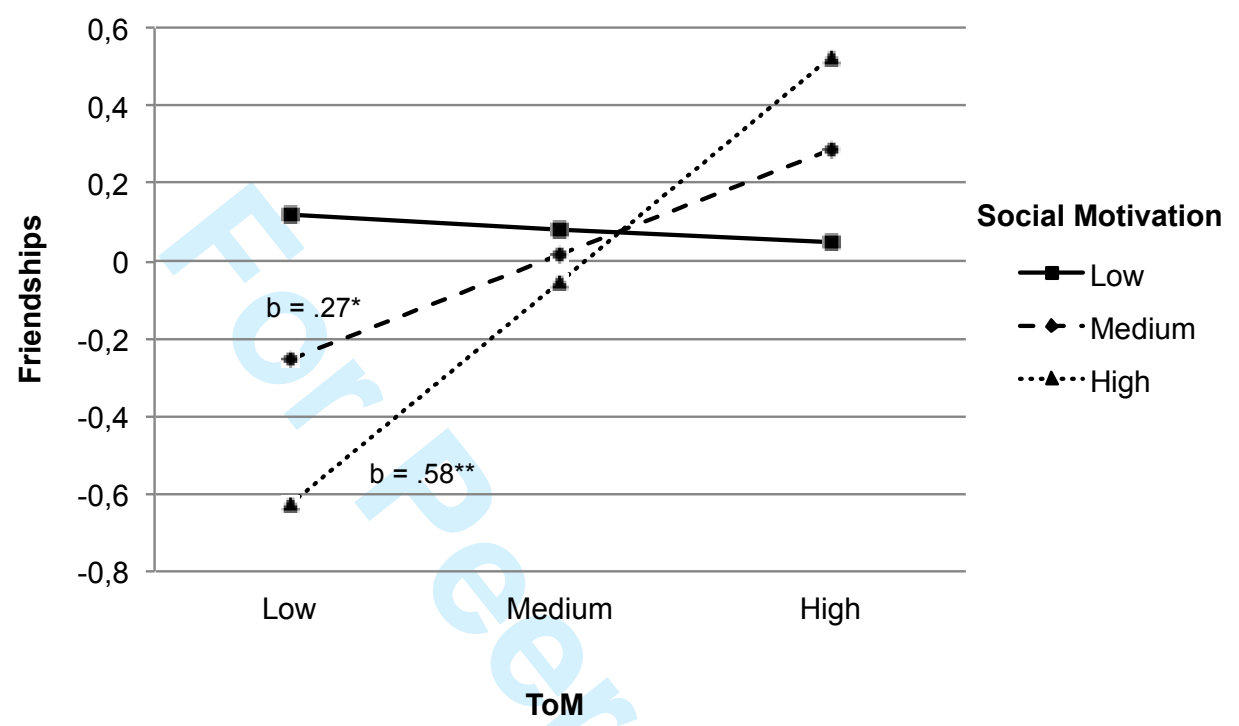

Note. Unstandardized beta coefficients are reported for simple slopes.

${ }^{+} p<.10 ; * p<.05 ; * * p<.01$ 